\title{
Benefits of Losartan in the Presence of Ovarian Cáncer
}

\author{
Mirta D Ambra* \\ University Buenos Aires and Member World Virology Society, Argentina
}

*Corresponding author: Mirta D Ambra, University Buenos Aires and Member World Virology Society, Argentina.

Received Date: November 25, 2019

Published Date: December 03, 2019

\section{Mini Review}

Losartan is an antihypertensive drug that acts on the tumor extracellular matrix. Its use can improve the arrival and action of chemotherapists in the presence of ovarian cáncer.

\section{Introduction and Objectives}

In most cases, the initial treatment of ovarian cancer patients consists of cytoreduction surgery and, subsequently, the application of chemotherapy with carboplatin or cisplatin and paclitaxel or docetaxel. Despite the initial response to treatment, many patients will have relapses and resistance. Due to the low rates of response to treatment, it is necessary to have alternatives. Drugs used in cancer patients must reach cancer cells; This can take place through the bloodstream and the subsequent passage of drugs through the vessel wall and interstitial tissue to reach the tumor. In desmoplastic malignant carcinomas there is a high level of solid stress caused by tumor and stromal cells and the fibrotic extracellular matrix. Solid stress is a physical force that compresses tumor blood vessels, with the consequent reduction in blood flow. This hinders the distribution of drugs and the effectiveness of treatment, in addition to generating resistance due to local hypoxia. The renin-angiotensin system (ARS) participates in the maintenance of cardiovascular homeostasis and hydroelectrolytic balance. In patients with ovarian carcinoma, an increase in the serum level of the angiotensin-converting enzyme (RCT), responsible for the generation of bioactive angiotensin II, was observed. An association was also found between the increased expression of angiotensin II type 1 receptor (AT1) and unfavorable evolution, and an inverse correlation between the level of tumor fibrosis and patient survival. Losartan is an antihypertensive drug that blocks the AT1 receptor. As found in experimental animals, the drug decreases intratumoral expression of thrombospondin-1(THBS-1), an activator of fibrinogenic beta (TGF-beta) tumor growth factor. This is associated with decreased collagen and intratumoral hyaluronan. As a result, reduction of solid stress and vessel compression, and increased vascular perfusion were observed. This resulted in a decrease in tumor hypoxia and an increase in the distribution and efficacy of drugs. The authors of the present study evaluated whether the administration of losartan, by reducing fibrosis of tumor tissue in patients with ovarian cancer, improves the distribution and efficacy of chemotherapeutic drugs administered by different routes.

\section{Methods}

The effect of losartan was retrospectively evaluated in two orthotopic ovarian cancer models, in stages IIIC or IV. Tumor cells were implanted in the peritoneal cavity of mice. Tumor growth was assessed by genetic analysis. Then, the mice were divided to receive losartan or to integrate the control group.

\section{Result}

Losartan treatment was associated with a significant decrease in extracellular matrix content in ovarian tumors. Specifically, a decrease in collagen and hyaluronan levels and fibrogenic fibroblast levels was observed. There was also a reduction in the expression of matrix molecules, although the expression of the main fibrogenic genes did not decrease significantly. Through antifibrotic effects, drug exposure was associated with the decompression of the vessels by decreasing solid stress and stiffness of the extracellular matrix. Losartan treatment was also correlated with improved perfusion and decreased tumor hypoxia. The modification of vessel density was not observed, but the proportion of perfused vessels increased and, consequently, hypoxia of viable tissue significantly decreased. The administration of losartan increased the distribution of chemotherapeutics to tumor tissue, thanks to the decompression of the vessels and increased perfusion. The quantitative analysis carried out allowed confirming that losartan significantly increased the level of intratumoral doxorubicin. Likewise, the application 
of a mathematical model allowed us to observe an increase in the distribution of the drug and an improvement in the efficacy chemotherapy in combination with losartan. According to the results of the applied model, treatment with losartan decreases the pressure of the interstitial fluid, increases the distribution of oxygen and improves the intratumoral distribution of chemotherapeutic drugs. This generates a decrease in tumor volume. Furthermore, it was observed that the increase in the diffusion of the drug or the decrease in solid stress increases the intratumoral distribution of the drug considerably. In ovarian cancer models, treatment with losartan increased the effectiveness of chemotherapeutics by modifying the extracellular matrix and blood vessels. This was observed when comparing treatment with losartan, paclitaxel, both drugs or placebo.

In addition, the complementary use of losartan was associated with a decrease in the incidence and level of ascites. Another effect of losartan was the accentuation of the decrease in proliferating and apoptotic tumor cells generated by paclitaxel. Losartan use was linked to the decrease in collagen content and normalization of the tumor lymphatic network. This suggests the reduction of solid stress. Another effect of losartan was the improvement of the function of the lymphatic vessels, reflected in the drainage and the clearance of a liquid with contrast. The induction of antifibrotic microRNA expression by losartan was also observed. In this sense, there was an increase in the expression of the miR-133 factor, which binds to the gene that codes for collagen type I ColIA1, with the decrease in the level of type I collagen in ovarian cancer cells. The use of an ACE inhibitor or an angiotensin receptor blocker was associated with increased survival in patients with advanced ovarian cancer. Retrospective analysis of women with stage IIIC or IV ovarian cancer treated at Massachusetts General Hospital (MGH) and at Brigham and Women's Hospital (BWH) indicated a median survival of 63 months before complementary treatment with these drugs. In contrast, women who did not receive such treatment had a median survival of 33 months. In addition, therapy with an ACE inhibitor was superior, compared to the use of an angiotensin receptor blocker.

\section{Discussion}

The treatment of patients with ovarian cancer involves difficulties related to diagnosis in advanced stages and resistance to chemotherapeutic agents. The results obtained indicate that blocking AT1 receptors limits extrinsic chemoresistance, controls ascites and increases survival. In addition, the analysis of fibrogenic microRNAs can serve as an indicator of chemotherapy resistance in patients with ovarian cancer. The findings indicated that the AT1 block decreases solid stress and improves the distribution of drugs to tumor tissue. The use of losartan and other angiotensin II type 1 receptor blockers is common in hypertensive patients. Losartan has good distribution and tissue penetration. Although this drug had no antitumor effects, it improved the distribution of chemotherapeutics by reducing solid stress and extracellular matrix content. These mechanisms indicate the importance of the evaluation of the clinical use of losartan to improve the efficacy of chemotherapeutic agents. Ascites is common in patients with stage II or IV ovarian cancer. So far, there are no effective treatments for the lasting control of ascites. Losartan reduced ascites by decreasing the tumor content of extracellular matrix. This could translate into improved quality of life. It is necessary to have additional studies in this regard. Treatment with an ACE inhibitor or an angiotensin receptor blocker was associated with an average survival increase of 30 months. However, the use of an angiotensin receptor blocker was superior. It should be considered that the analysis was retrospective, with which there are confounding factors that limit the reliability of the results. In any case, the findings obtained justify the performance of additional studies that allow corroborating the benefits of the treatment [1].

\section{Conclusion}

The results obtained in the present study allowed us to demonstrate that the decrease in the extracellular matrix in ovarian cancer models is associated with an improvement in the efficacy of chemotherapy and a decrease in the level of ascites. content and normalization of the tumor lymphatic network. This suggests the reduction of solid stress. Another effect of losartan was the improvement of the function of the lymphatic vessels, reflected in the drainage and the clearance of a liquid with contrast. The induction of antifibrotic microRNA expression by losartan was also observed. In this sense, there was an increase in the expression of the miR-133 factor, which binds to the gene that codes for collagen type I ColIA1, with the decrease in the level of type I collagen in ovarian cancer cells. The use of an ACE inhibitor or an angiotensin receptor blocker was associated with increased survival in patients with advanced ovarian cancer. Retrospective analysis of women with stage IIIC or IV ovarian cancer treated at Massachusetts General Hospital (MGH) and at Brigham and Women's Hospital (BWH) indicated a median survival of 63 months before complementary treatment with these drugs. In contrast, women who did not receive such treatment had a median survival of 33 months. In addition, therapy with an ACE inhibitor was superior, compared to the use of an angiotensin receptor blocker.

\section{Acknowledgment}

None.

\section{Conflict of Interest}

No conflict of interest.

\section{References}

1. Zhao Y, Cao J, Melamed A, Worley M, Gockley A, et al. (2019) Losartan treatment enhances chemotherapy efficacy and reduces ascites in ovarian cancer models by normalizing the tumor stroma. Proc Natl Acad Sci USA 116 (6): 2210- 2219. 